Relations industrielles

Industrial Relations

\title{
Von Prondzynski : Freedom of Association and Industrial Relations. London, Mansell Publishing, 1987, 248 pp., ISBN 0-7201-1775-5
}

\section{Pierre Verge}

Volume 42, numéro 3, 1987

URI : https://id.erudit.org/iderudit/050345ar

DOI : https://doi.org/10.7202/050345ar

Aller au sommaire du numéro

\section{Éditeur(s)}

Département des relations industrielles de l'Université Laval

\section{ISSN}

0034-379X (imprimé)

1703-8138 (numérique)

Découvrir la revue

Citer ce compte rendu

Verge, P. (1987). Compte rendu de [Von Prondzynski : Freedom of Association and Industrial Relations. London, Mansell Publishing, 1987, 248 pp., ISBN 0-7201-1775-5]. Relations industrielles / Industrial Relations, 42(3), 651-652. https://doi.org/10.7202/050345ar

Tous droits réservés (C) Département des relations industrielles de l'Université Laval, 1987
Ce document est protégé par la loi sur le droit d'auteur. L’utilisation des services d’Érudit (y compris la reproduction) est assujettie à sa politique d'utilisation que vous pouvez consulter en ligne.

https://apropos.erudit.org/fr/usagers/politique-dutilisation/ 
Overall, this book presents a comprehensive theory of union mergers that is capable of explaining the forces acting for and against mergers, as well as the characteristics of, and links between the pre- and postmerger states. It is thought-provoking and carefully researched. It is a valuable resource for researchers and students interested in unions in North America. Chaison's lucid and rich writing style makes it interesting to read about the complex process of union mergers.

Till now, there was no unifying or comprehensive theory of union merger process despite the recognition of the past and future importance of union mergers. Chaison's book fills this void by providing a model with ability to explain more than the contemporary mergers. It is the most definitive work on union mergers and I recommend it to any scholar interested in the study of unions.

P. ANDIAPPAN

University of Windsor

Freedom of Association and Industrial Relations, by F. von Prondzynski, London, Mansell Publishing Limited, 1987, 248 pp., ISBN 0-7201-1775-5

Le législateur et le juge oscillent, selon les pays et les époques, entre deux grandes conceptions de la liberté syndicale: d'une part, dans une perspective politico-libérale, la faculté de l'individu de s'associer ou de s'abstenir de le faire, faculté qui ne souffre que quelques exceptions, en raison, notamment, de la sécurité publique; de l'autre, une notion «fonctionnelle» ou téléologique du recours à l'action syndicale; on l'assure alors en tant que moyen d'atteindre un certain ordre social, plus spécifiquement, un équilibre du pouvoir de négociation entre employeurs et salariés. Moins nette que la première, cette seconde notion n'est pas moins importante; elle exprime les préalables économiques d'une liberté significative et touche ainsi à la répartition du pouvoir dans la société.

L'objet de l'auteur est d'établir dans quelle mesure l'ordre juridique de certains pays reflète l'une ou l'autre de ces conceptions de la liberté syndicale. La préoccupation vise primordialement le Royaume-uni (a l'exclusion de l'Irlande du Nord) et la République d'Irlande, mais elle conduit aussi à des comparaisons avec la situation en République fédérale allemande, de même qu'aux États-Unis. Si les pages sur lesquelles se referme le livre dénoncent le retrait qu'opèrent les interventions législatives des récentes années du gouvernement de madame Thatcher dans le domaine des relations du travail par rapport à cette seconde conception fonctionnelle de la liberté syndicale, l'ensemble de l'ouvrage envisage le sujet avec le recul historique nécessaire. L'auteur, présentement lecturer en relations industrielles à Trinity College, Dublin s'appuie en partie sur une précédente thèse de doctorat.

Indépendamment de la dizaine de chapitres qui le composent, l'ouvrage paraît comporter essentiellement deux plans. La première moitié, qui regroupe les six premiers chapitres, présente une analyse générale de la liberté syndicale. D'abord, quelques pages distinguent, dans le sillage du juriste américain Hohfeld, le concept de droit de celui de liberté. En fait, l'intervention législative, qui protège la liberté d'association à l'endroit de tiers, crée autant de «droits», au-delà de la simple permissivité étatique à la quelle se réduirait autrement la liberté d'association. Cet exposé initial précède une brève typologie des grands modes de régulation de l'action syndicale dans les pays en cause. Jusqu'où en définitive, un état peut-il favoriser certains syndicats, en leur conférant par exemple, un monopole de représentation, comme aux Etats- 
Unis, voire, une exclusivité de membership dans un secteur professionnel particulier? La notion fonctionnelle de liberté d'association devrait aussi, par ailleurs, conduire l'État à assurer l'indépendance des instances syndicales: ainsi, la loi fondamentale de la R.F.A. interdit-elle l'ingérence patronale dans les affaires syndicales; par contre, on ne trouve pas de prohibitions législatives équivalentes en Angleterre. De même, l'exercice de la liberté syndicale par les salariés suppose-t-elle la protection individuelle de ceux-cì contre de possibles représailles de l'employeur: corollaire de la reconnaissance constitutionnelle de l'aspect collectif de la liberté d'association en Allemagne; pratiques déloyales de la loi fédérale américaine. L'intérêt collectif du groupement doit aussi être protégé. Il y va de l'assurance, pour les syndicats de pouvoir s'engager dans des négociations collectives significatives. Mais, l'État doit-il aller jusqu'à imposer la reconnaissance syndicale aux employeurs? L'absence d'une telle obligation de reconnaissance en droit anglais porte l'auteur à douter (p. 102) de la conformité de celui-ci aux engagements internationaux du pays en cause. Enfin, question d'une grande actualité pour le lecteur canadien, à la suite des arrêts de la Cour suprême du Canada du 9 avril 1987 sur le sujet, au regard de l'article 2d) de la Charte des droits et libertés: le droit de grève est-il compris dans l'affirmation de la liberté syndicale? La conception fonctionnelle de la liberté d'association entraîne une réponse affirmative, l'action syndicale, pour être significative, doit comporter un pouvoir de sanction: "At the collective level of industrial relations it is hard to envisage freedom of association without a freedom to strike» (p. 109). La réponse de la majorité du banc de la plus haute instance procède de l'autre acception de la liberté d'association pour les fins, à tout le moins, de l'interprétation constitutionnelle. Ces pages pourront contribuer à bien dégager la véritable portée de cet arrêt.

Le second volet de l'ouvrage - ses quatre derniers chapitres - est centré sur une application particulière des deux grandes conceptions précédentes de la liberté syndicale: l'atelier syndical. Le sujet nécessite d'abord de s'interroger - ce qui serait particulièrement valable en contexte canadien - sur la nature de l'instance syndicale contemporaine: elle exerce des fonctions publiques, comme l'avait déjà posé Kahn-Freund. Dans cette perspective, l'appartenance syndicale, condition d'emploi, s'apparente a un tribut; en retour, le syndicat ne peut plus pratiquer des comportements particuliers en matière d'appartenance syndicale. Des exceptions sont aussi envisageables en faveur de certains objecteurs. Cette prise de position sert en quelque sorte d'arrière-plan à l'examen successif de la licéité de l'atelier syndical au regard de la Common Law - acceptation graduelle d'une justification de la coalition en fonction de la nature de l'intérêt poursuivi -, de l'intervention législative - une réglementation de plus en plus serrée en Angleterre, à la suite, notamment du Employment Act 1982 -, et, enfin, de l'arrêt de 1981 de la Cour européenne des Droits de l'Homme dans Young, James and Webster. Dans cette affaire célèbre, la majorité des juges, défavorable à l'obligation d'adhérer au syndicat sous peine de perte d'emploi imposée à des salariés déjà en place, n'aurait-elle pas transformé une simple liberté de ne pas appartenir à un syndicat, qui proscrit la contrainte légale pour forcer l'appartenance syndicale, en un véritable droit, opposable à des tiers, de ne pas appartenir au syndicat?

Cette question, qui permet à nouveau à l'auteur de manier ses concepts initiaux, n'est pas non plus dépourvue d'intérêt dans le contexte des chartes canadienne et québécoise. Dans la perspective comparatiste qu'il a adoptée, elle se rattache également à cette démarche, plus générale et fondamentale, de définition de la compréhension de la liberté syndicale à partir de sa fonction sociale.

Pierre VERGE 